\title{
Resveratrol inhibits the growth of tumor cells under chronic stress via the ADRB-2-HIF-1 $\alpha$ axis
}

\author{
JIGUANG MA $^{1 *}$, MENGWEN XUE $^{1 *}$, SIMEI ZHANG ${ }^{1}$, LIANG CHENG $^{2}$, \\ WEIKUN QIAN ${ }^{2}$, WANXING DUAN ${ }^{2}$ and XIN SHEN ${ }^{1}$ \\ Departments of ${ }^{1}$ Anesthesiology and ${ }^{2}$ Hepatobiliary Surgery, First Affiliated Hospital of Medical College, \\ Xi'an Jiaotong University, Xi'an, Shaanxi 710061, P.R. China
}

Received February 14, 2018; Accepted November 14, 2018

DOI: $10.3892 / o r .2018 .6894$

\begin{abstract}
Resveratrol is a type of polyphenol that is abundantly present in knotweed and grapes, and has been confirmed to have tumor-inhibitory properties. However, the effect of resveratrol on tumor cells under chronic stress conditions remains unclear. The aim of the present study was to demonstrate that resveratrol exerts a significant inhibitory effect on the growth and proliferation of tumor cells under chronic stress in a dose-dependent manner. Furthermore, resveratrol was able to induce apoptosis of cancer cells under chronic stress conditions. Moreover, resveratrol was found to inhibit tumor cells under chronic stress by decreasing the expression of the $\beta_{2}$-adrenergic receptor (ADRB-2); in addition, the protein expression of hypoxia-inducible factor (HIF)- $1 \alpha$ was suppressed by resveratrol in a dose-dependent manner. Thus, these data suggest that resveratrol inhibits the growth of cancer cells under chronic stress by regulating the ADRB-2-HIF-1 $\alpha$ axis. In summary, the present study may provide a new basis supporting the use of resveratrol for the prevention and treatment of pancreatic cancer.
\end{abstract}

\section{Introduction}

Cancer is the second cause of death worldwide, with increasing incidence and mortality rates, and it is the leading cause

Correspondence to: Professor Xin Shen, Department of Anesthesiology, First Affiliated Hospital of Medical College, Xi'an Jiaotong University, 277 West Yanta Road, Xi'an, Shaanxi 710061, P.R. China E-mail: shenxin6125@mail.xjtu.edu.cn

Dr Wanxing Duan, Department of Hepatobiliary Surgery, First Affiliated Hospital of Medical College, Xi'an Jiaotong University, 277 West Yanta Road, Xi'an, Shaanxi 710061, P.R. China

E-mail: 15339184808@163.com

"Contributed equally

Key words: chronic stress, resveratrol, $\beta_{2}$-adrenergic receptor, hypoxia-inducible factor- $1 \alpha$, cancer development of death in China. Data from the National Central Cancer Registry of China indicate that an estimated 3,682,000 new cancer cases and 2,229,300 cancer-related deaths occurred in 2013 in China, and that 4,292,000 new cancer cases and 2,814,000 cancer deaths occurred in 2015 (1,2). An increasing number of studies have reported that social and psychological factors are implicated in the process of cancer development, mainly through pathophysiological mechanisms triggered in response to stress (3). Therefore, the effect of chronic stress on cancer patients should not be overlooked.

Currently available studies provide convergent evidence that two pathways, namely the hypothalamic-pituitary-adrenal (HPA) axis and the sympathetic nervous system (SNS), are activated under chronic psychological stress conditions. The $\beta_{2}$-adrenergic receptor (ADRB-2), a SNS receptor, which can be activated by chronic stress, contributes to the tumorigenesis and cancer development. Hypoxia-inducible factor (HIF)- $1 \alpha$ expression is induced by lack of oxygen, which has been found to be closely associated with the occurrence and development of several types of tumors. Our previous study revealed that activation of the ADRB-2/HIF-1 $\alpha$ pathway plays an essential role in cancer growth and angiogenesis under chronic stress conditions (4). Thus, identifying a treatment targeting the ADRB-2/HIF-1 $\alpha$ axis may serve as an effective strategy for cancer therapy.

Resveratrol (Res) is a type of polyphenol abundantly present in knotweed and grapes, and it has been confirmed to inhibit tumor development (5-8). The mechanism underlying the anticancer properties of Res has been extensively investigated, and it includes metabolic shift (9), inhibition of migration and invasion of cancer cells (10), induction of autophagy (11), as well as direct cytotoxicity (12). However, the effect of Res on cancer cells under chronic stress remains unclear. The aim of the present study was to determine whether Res inhibits tumor cell proliferation and promotes apoptosis via suppressing catecholamine neurotransmitters induced by the ADRB-2/HIF- $1 \alpha$ axis activation in cancer.

\section{Materials and methods}

Cell culture and major reagents. The BxPC-3 human pancreatic cancer cell line was purchased from the Cell Bank of the Chinese Academy of Sciences (Shanghai, China). The human 
hepatoblastoma HepG2 cell line (13) was generously provided by Dr Chang Liu (Medical College, Xi'an Jiaotong University). The SKOV-3 ovarian carcinoma cell line was kindly provided by the First Affiliated Hospital Obstetrics and Gynecology Laboratory at Xi'an Jiaotong University. BxPC-3 and HepG2 cells were cultured in DMEM, and SKOV-3 cells were cultured in RPMI-1640 medium. The medium was supplemented with $10 \%$ fetal bovine serum (FBS), and cells were maintained under normal culture conditions of $5 \% \mathrm{CO}_{2}$ at $37^{\circ} \mathrm{C}$. Res, norepinephrine (NE), HIF-1 $\alpha$ antibody (diluted 1:1,000, ab51608), ADRB-2 antibody (diluted 1:1,000, ab61778) and MTT were purchased from Sigma-Aldrich; Merck KGaA (Darmstadt, Germany). Proliferating cell nuclear antigen (PCNA) and Ki-67 antibodies (both diluted 1:200, sc-56 and sc-15402) were obtained from Santa Cruz Biotechnology, Inc. (Santa Cruz, CA, USA).

MTT cell viability assay. BxPC-3, HepG2 and SKOV-3 cancer cells were plated in 96-well plates at a density of $5 \times 10^{3}$ cells/ well and treated with $10 \mu \mathrm{M}$ norepinephrine (NE). After $24 \mathrm{~h}$, the NE-containing medium was removed and various concentrations $(0,25,50,75$ and $100 \mu \mathrm{M})$ of Res were added to the cells. Cell viability was assessed by the MTT assay at the indicated time points (24, 36 and $48 \mathrm{~h})$. A multi-well microplate reader (BIO-TEC, Inc., Richmond, VA, USA) was used to measure the absorbance at $490 \mathrm{~nm}$.

Colony formation assay. Cells (1,000 cells/well) were seeded in triplicate and allowed to adhere overnight, and they were then incubated with NE for $48 \mathrm{~h}$. Subsequently, the cells were treated with Res without NE-containing medium for $24 \mathrm{~h}$ followed by incubation with media lacking Res for 7-10 days. The cells were then fixed with $4 \%$ paraformaldehyde and stained with $0.1 \%$ crystal violet solution, and the colonies ( $>50$ cells) were counted.

Apoptosis assay. Apoptosis was determined by flow cytometry with an Annexin V-FITC/PI apoptosis detection kit (Beyotime Institute of Biotechnology, Shanghai, China). Briefly, cells were seeded at density of $1 \times 10^{5}$ cells/well in 6-well plates and cultured to the indicated density followed by treatment with $10 \mu \mathrm{M} \mathrm{NE}$ for $24 \mathrm{~h}$. Cells were treated with fresh medium containing various concentrations $(0,25$ and $50 \mu \mathrm{M})$ of Res for an additional $24 \mathrm{~h}$. Subsequently, the cells were trypsinized, washed with phosphate-buffered saline (PBS) and stained with Annexin V and PI. Apoptosis was evaluated using flow cytometry (FACSCalibur; BD Biosciences, Franklin Lakes, NJ, USA).

Western blotting. For analysis of the HIF-1 $\alpha$, ADRB-2, proliferating cell nuclear antigen (PNCA) and $\mathrm{Ki}-67$ protein expression, cell lysates were prepared from BxPC-3, HepG2 and SKOV-3 cells, which had been treated with medium containing $10 \mu \mathrm{M} \mathrm{NE}$ for $24 \mathrm{~h}$ followed by treatment with fresh medium containing various concentrations $(0,25$ and $50 \mu \mathrm{M})$ of Res. Total proteins were extracted using RIPA lysis buffer (Sigma Aldrich; Merck KGaA), separated by $10 \%$ sodium dodecyl sulfate polyacrylamide gel electrophoresis and transferred onto PVDF membranes. After blocking with 5\% non-fat milk in Tris-buffered saline Tween-20 (TBST) for

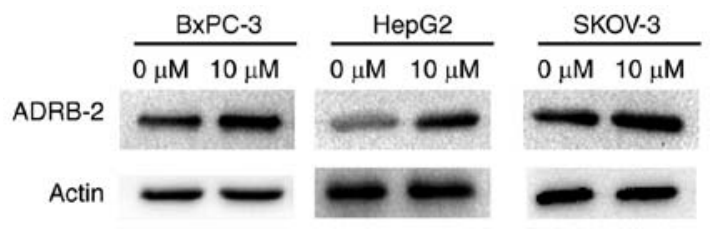

Figure 1. NE treatment increases the expression of ADRB-2 in BxPC-3, HepG2 and SKOV-3 cancer cell lines. Western blot analysis demonstrated that ADRB-2 expression was enhanced in the three cell lines following treatment with NE. NE, norepinephrine; ADRB-2, $\beta_{2}$-adrenergic receptor.

$1 \mathrm{~h}$, the membranes were incubated with primary antibody overnight at $4^{\circ} \mathrm{C}$. The primary antibody was removed, and the membranes were then incubated with 1:2,000 horseradish peroxidase-conjugated secondary antibody for $2 \mathrm{~h}$ at room temperature. Protein expression was visualized with enhanced chemiluminescence, and images were captured using the ChemiDoc XRS imaging system (Bio-Rad Laboratories, Inc., Hercules, CA, USA). Quantity One image software was used for the densitometry analysis of each band. $\beta$-actin was used as an internal loading control.

Reverse transcription-quantitative polymerase chain reaction (RT-qPCR) analysis. Total cell RNA was extracted using TRIzol reagent following the manufacturer's instructions (Invitrogen; Thermo Fisher Scientific, Inc., Waltham, MA, USA). cDNA synthesis was performed using a PrimeScript RT reagent kit (Takara Biotechnology Co., Ltd., Dalian, China). qPCR was performed with an iQ5 Multicolor Real-Time PCR Detection System (Bio-Rad Laboratories, Inc.) using a SYBR-Green PCR kit (Takara Biotechnology Co., Ltd.) according to the manufacturer's instructions. The amplification consisted of predenaturation at $95^{\circ} \mathrm{C}$ for $5 \mathrm{~min}$, denaturation at $95^{\circ} \mathrm{C}$ for $30 \mathrm{sec}$, annealing at $58^{\circ} \mathrm{C}$ for $30 \mathrm{sec}$ and extension at $72^{\circ} \mathrm{C}$ for $30 \mathrm{sec}$ for 32 cycles. After the last cycle, a final extension was performed at $72^{\circ} \mathrm{C}$ for $7 \mathrm{~min}$. The primer sequences for ADRB-2 were as follows: Forward 5'-GGG TCTTTCAGGAGGCCAAA-3' and reverse 5'-ATGCCTAAC GTCTTGAGGGC-3'; the primer sequences for HIF-1 $\alpha$ were as follows: Forward 5'-CTTGGCAACCTTGGATTGGAT G-3' and reverse 5'-AATCTCCGTCCCTCAACCTCT-3'. The $\Delta \Delta \mathrm{Cq}$ method was used to calculate the relative expression of the sample genes with $\beta$-actin as the normalized reference gene.

Immunofluorescence staining. Cells were plated in chamber slides, incubated for $24 \mathrm{~h}$, and then treated as indicated. The cells were fixed using $4 \%$ paraformaldehyde for $15 \mathrm{~min}$ and permeabilized using PBS containing $0.5 \%$ Triton X-100 for $20 \mathrm{~min}$ at room temperature. The cells were then incubated with the primary antibody overnight at $4^{\circ} \mathrm{C}$, followed by incubation with fluorescein-conjugated secondary antibody at room temperature for $1 \mathrm{~h}$. The cells were then visualized using a fluorescence microscope.

Statistical analysis. Each experiment was performed at least three times. The results are expressed as the mean \pm standard deviation of the number of experiments. One-way analysis of variance was used to evaluate the statistical significance between groups. P-value $<0.05$ was considered to indicate 
A

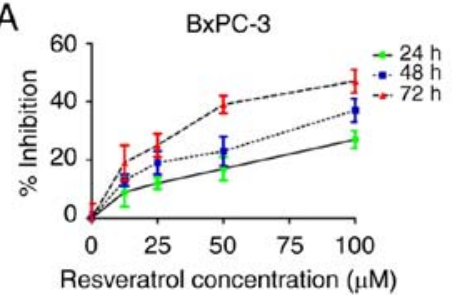

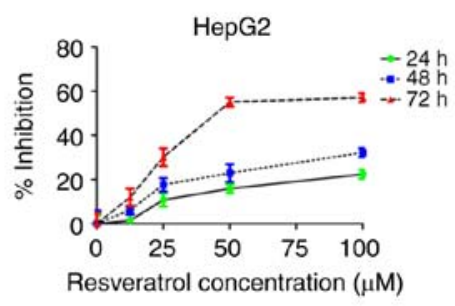

Resveratrol concentration $(\mu \mathrm{M})$

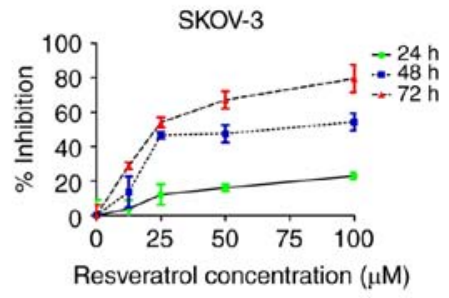

B

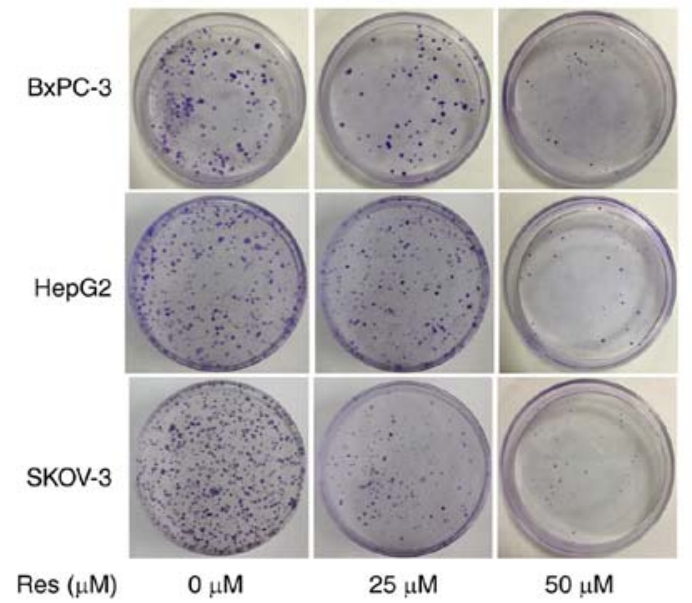

D

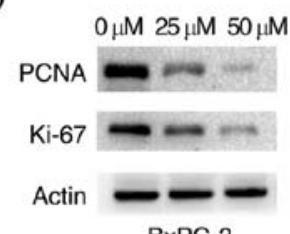

BxPC-3
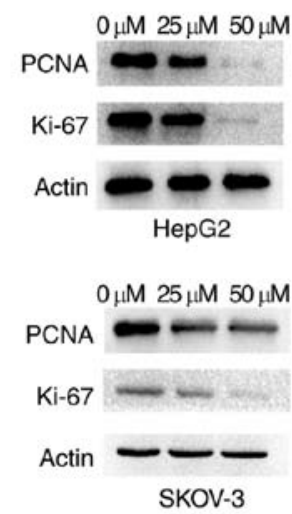

Figure 2. Inhibitory effect of Res on the proliferation of chronically stressed cancer cells. (A) Chronically stressed cancer cells (BxPC-3, HepG2 and SKOV-3) were treated with different concentrations of Res $(0,25,50,75$ and $100 \mu \mathrm{M}$ ). After 24, 48 and 72 h, cell viability was assessed by an MTT assay. (B and C) Res treatment inhibited the colony-forming ability of chronically stressed BxPC-3, HepG2 and SKOV-3 cancer cells (*P<0.05). (D) PNCA and Ki-67 protein expression was detected in the chronically stressed BxPC-3, HepG2 and SKOV-3 cancer cells. Treatment of the three chronically stressed cancer cell lines with 25 and $50 \mu \mathrm{M}$ Res significantly reduced the expression of PNCA and Ki-67. Res, resveratrol; PNCA, proliferating cell nuclear antigen.

statistically significant differences. All statistical analyses were performed using SPSS software, version 15.0 (SPSS, Inc., Chicago, IL, USA).

\section{Results}

$N E$ induces the expression of $A D R B-2$ in $B x P C-3$, Hep $G 2$ and $S K O V-3$ cancer cell lines. To imitate a chronic stress condition in vitro, three cell lines, namely BxPC-3, HepG2 and SKOV-3, were treated with NE. After $24 \mathrm{~h}$, western blotting was used to detect ADRB-2 expression. The results demonstrated that ADRB-2 expression was significantly increased after treatment, indicating that NE may be used for in vitro experiments to simulate chronic stress (Fig. 1).

Res inhibits the proliferation of cancer cells under chronic stress conditions. First, the effects of Res on the viability of chronically stressed cancer cells were examined. Res inhibited chronically stressed cancer cells in a dose- and time-dependent manner (Fig. 2A). Colony formation ability was detected after the addition of Res to chronically stressed cancer cells. Compared with untreated cells, treatment with $25 \mu \mathrm{M}$ Res markedly decreased the number of colonies, and there was almost no colony formation when the concentration of Res was increased to $50 \mu \mathrm{M}(\mathrm{P}<0.05)$ (Fig. 2B). PCNA is located in the nucleus and plays an important role in cell proliferation. $\mathrm{Ki}-67$ protein expression is used as a marker to indicate cell proliferation. To test whether Res inhibits the proliferation of chronically stressed cancer cells, PCNA and Ki-67 protein expression was measured in the three cell lines. Western blot analysis revealed that $\operatorname{Res}(0,25$ and $50 \mu \mathrm{M})$ suppressed PCNA and $\mathrm{Ki}-67$ expression in chronically stressed cancer cells in a dose-dependent manner (Fig. 2C).

Res induces apoptosis of chronically stressed cancer cells. To determine whether Res induces cancer cell apoptosis under chronic stress conditions, flow cytometric analyses were conducted after the cancer cells (BxPC-3, HepG2 and 

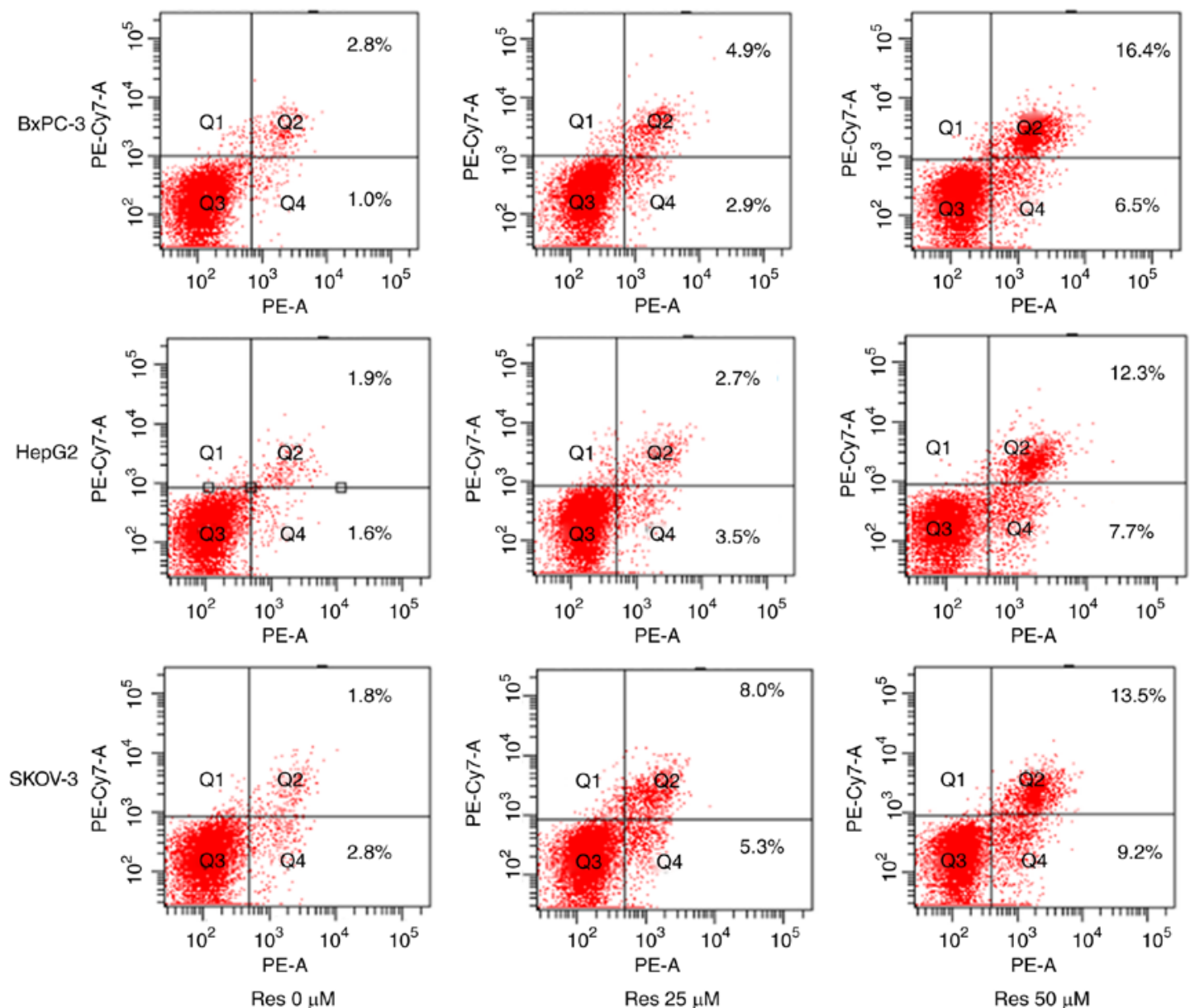

Res $25 \mu \mathrm{M}$

Res $50 \mu \mathrm{M}$
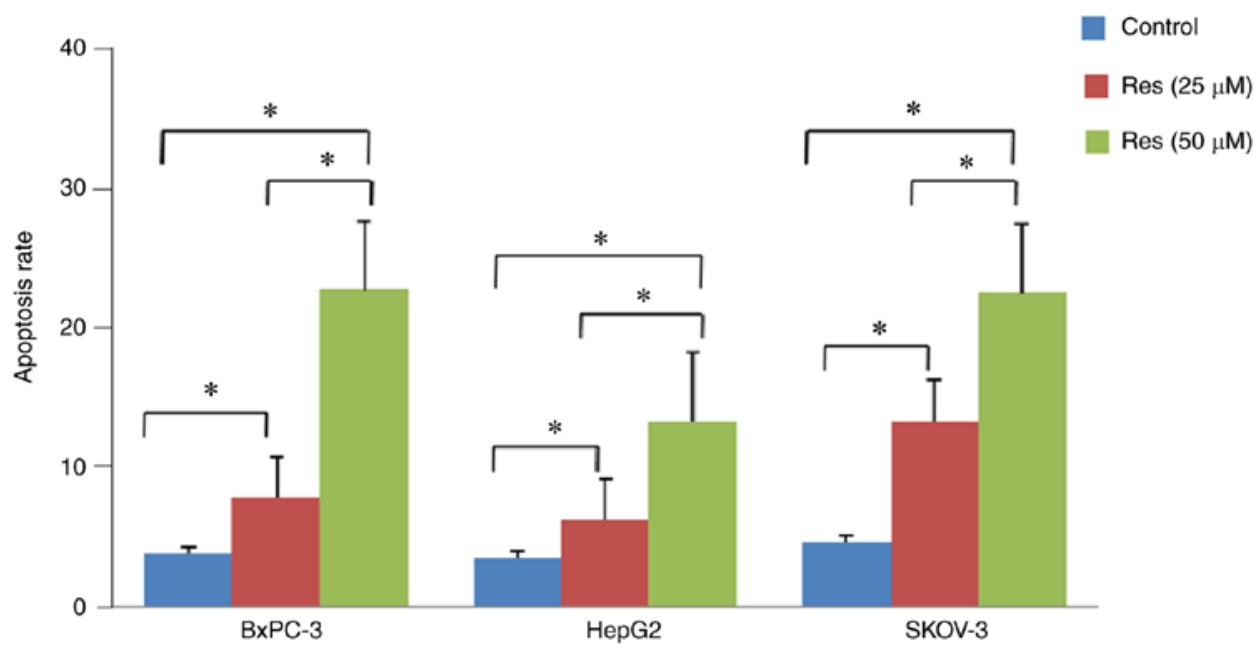

Figure 3. Res treatment induces apoptosis of chronically stressed cancer cells. After treatment of the three chronically stressed cancer cell lines with 0 , 25 and $50 \mu \mathrm{M}$ Res for $48 \mathrm{~h}$, flow cytometry was used to detect cancer cell apoptosis ( $\mathrm{P}<0.05)$. Res, resveratrol.

SKOV-3) were treated with Res $(25$ and $50 \mu \mathrm{M})$ for $48 \mathrm{~h}$ in the presence of NE. The apoptosis rates of the cancer cells were calculated by summing up the quadrants of the early (lower right) and late (upper right) apoptosis of the FITC graphs. Res increased apoptosis of the three chronically stressed cancer cell types compared with untreated cells (Fig. 3, P<0.05).

Res suppresses chronically stressed cancer cell proliferation through the ADRB-2/HIF-1 $\alpha$ axis. Previous studies provided evidence that ADRB-2 plays an important role in chronic stress-induced tumor growth (4). Based on our previous findings, we hypothesized that the inhibition of chronically stressed cancer cells by Res may be mediated by the ADRB-2/HIF-1 $\alpha$ axis. To verify the effect of Res on the ADRB-2/HIF-1 $\alpha$ axis, RT-qRCR, western blotting and immunofluorescence assays were used to measure ADRB-2 and HIF-1 $\alpha$ expression after treatment of chronically stressed BxPC-3, HepG2 and SKOV-3 cancer cells with 0,25 and $50 \mu \mathrm{M}$ Res. RT-qRCR demonstrated that Res treatment markedly decreased ADRB-2 and HIF-1 $\alpha$ expression at the mRNA level $(\mathrm{P}<0.05)($ Fig. 4A). 

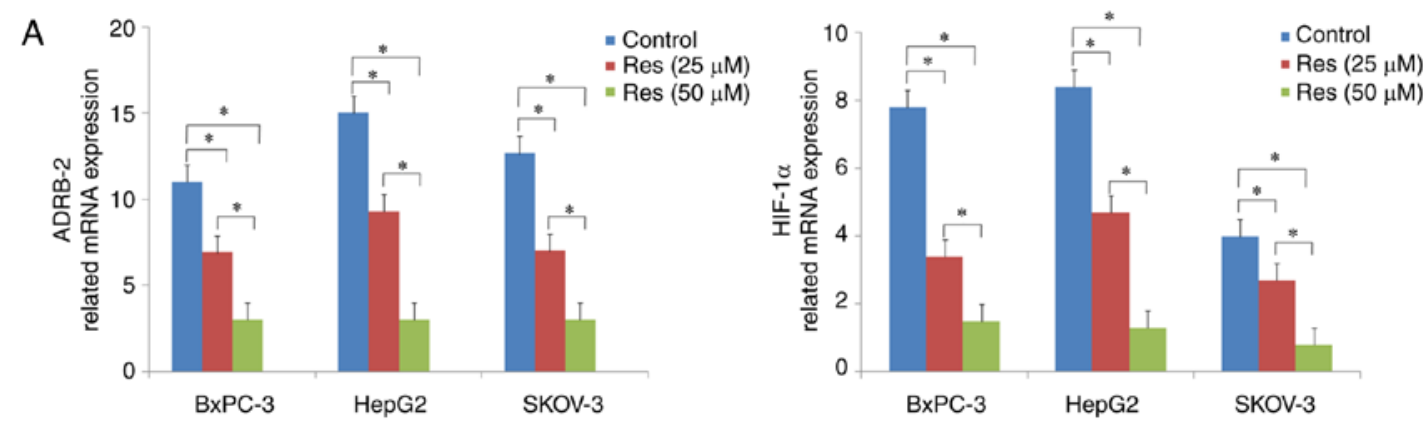

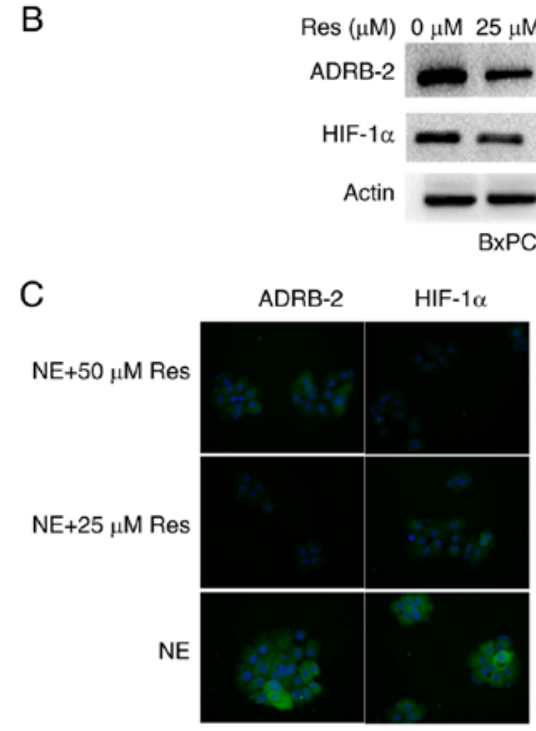

BxPC-3

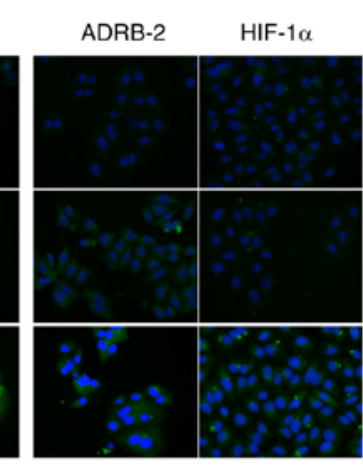

HepG2

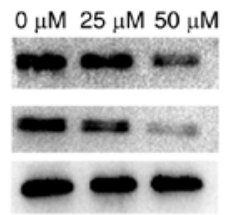

HepG2
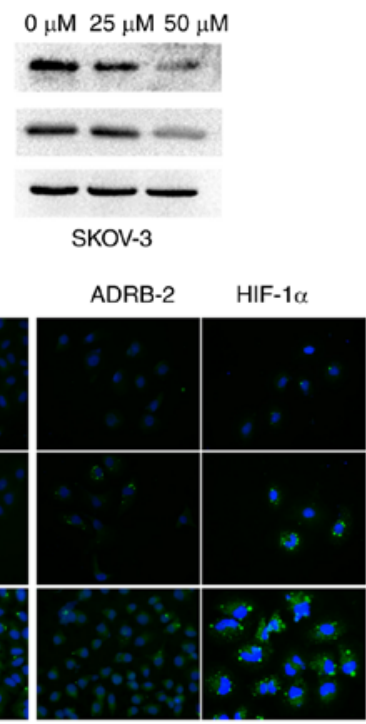

SKOV-3

Figure 4. Res suppresses the proliferation of cancer cells under chronic stress through the ADRB-2-HIF-1 $\alpha$ axis. (A) The effects of Res on the mRNA expression of ADRB-2 and HIF-1 $\alpha$ were examined by qPCR with $\beta$-actin as the normalized reference gene ( $\mathrm{P}<0.05)$. (B) The effects of Res on the protein expression of ADRB-2 and HIF-1 $\alpha$ were examined by western blotting. (C) Immunofluorescence detection of ADRB-2 and HIF-1 $\alpha$ protein in chronically stressed BxPC-3, HepG2 and SKOV-3 cancer cells treated with and without Res. Magnification, x200. Res, resveratrol; ADRB-2, $\beta_{2}$-adrenergic receptor; HIF-1 $\alpha$, hypoxia-inducible factor-1 $\alpha$; qPCR, quantitative polymerase chain reaction.

The protein expression levels of ADRB-2 and HIF-1 $\alpha$ were confirmed by western blotting (Fig. 4B). Consistently, the immunofluorescence results revealed that the ADRB-2 and HIF-1 $\alpha$ protein levels were markedly decreased following Res treatment (Fig. 4C). Taken together, these findings confirmed that Res inhibits chronically stressed cancer cell proliferation via inhibiting ADRB-2 and HIF-1 $\alpha$ expression.

\section{Discussion}

Chronic stress is characterized by inability to adjust to the circumstances or cope with emotional and psychological pressure. A number of studies have reported that chronic stress interferes with immune, endocrine and neural system functions. A number of epidemiological and clinical studies have reported that chronic stress plays an essential role in cancer occurrence, development and mortality, and reducing stress may prolong survival and decrease recurrence after therapy (14-17). It has been demonstrated that chronic stress can activate the HPA axis and the SNS, leading to a release of catecholamines (NE, epinephrine and dopamine), glucocorticoids and other hormones. NE, a stress hormone, has been found to stimulate cancer cell proliferation, invasion and metastasis through $\beta$-adrenergic receptors (18-20). In addition, our previous data demonstrated that HIF- $1 \alpha$ is a downstream target of ADRB, and that the ADRB-2-HIF-1 $\alpha$ axis induces cancer growth in animal stress models (4). Thus, the identification of drug targets within the ADRB-2-HIF-1 $\alpha$ axis may be of value in cancer therapy. Accumulating evidence has shown that Res exerts a potent inhibitory effect on tumor growth in pancreatic cancer (21), hepatoblastoma (22) and ovarian cancer (6). However, it remains unknown whether Res can inhibit tumor growth under chronic stress conditions. In the present study, Res was shown to inhibit cancer development and progression through the ADRB-2/HIF-1 $\alpha$ axis under chronic stress conditions.

HIF- $1 \alpha$ is a pivotal molecule and its activation has long been known to drive cancer cell responses to a hypoxic microenvironment by regulating the expression of genes involved in cell proliferation/survival, apoptosis, metastasis and resistance to radio- and chemotherapy $(23,24)$. However, HIF-1 $\alpha$ may also be expressed under normoxic conditions (4). Sun et al reported (25) that Res inhibited HIF-1 $\alpha$ protein accumulation without affecting the HIF-1 $\alpha$ mRNA level, which was not consistent with our results. Our study focused on chronically stressed cancer cells, and the three cell lines were treated with NE to simulate chronic stress conditions. The HIF- $1 \alpha$ mRNA levels in the three chronically stressed cancer cell lines were 

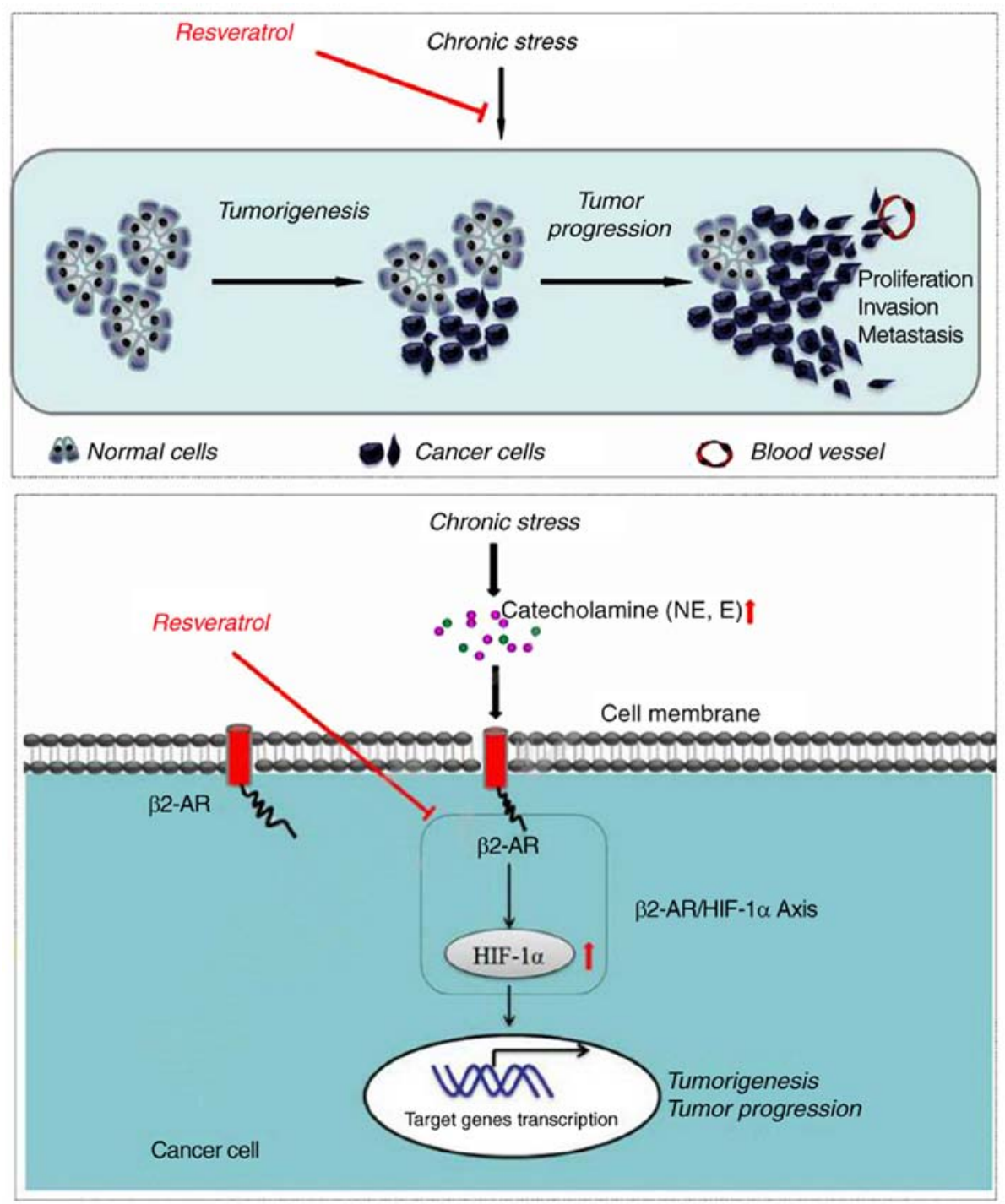

Figure 5. Resveratrol inhibits cancer progression under chronic stress conditions. Cancer cells subjected to chronic stress release catecholamines [norepinephrine (NE) and epinephrine (E)], which may promote cancer through the ADRB-2-HIF-1 $\alpha$ axis. After treatment with resveratrol, the chronically stressed cancer cells exhibited downregulation of ADRB-2 and HIF-1 $\alpha$ at the protein level, ultimately inhibiting tumor cell proliferation and inducing apoptosis. ADRB-2, $\beta_{2}$-adrenergic receptor; HIF-1 $\alpha$, hypoxia-inducible factor- $1 \alpha$.

found to be significantly inhibited by Res, although our results were not consistent with those of Sun et al (25). We inferred that NE may play an important role.

$\mathrm{NE}$ is a catecholamine, the secretion of which is increased during stress. Preclinical models have suggested that NE plays a decisive role in cancer promotion. Thus, NE treatment of cells or animals may mimic stress in in vitro and in vivo models $(26,27)$. Therefore, NE-treated cancer cells were used to mimic a chronic stress state, which allowed testing the effects of Res on chronically stressed cancer cells. First, MTT, colony formation and western blotting data revealed that Res inhibited chronically stressed cancer cell proliferation in a dose- and time-dependent manner. Cell apoptosis was assessed after the chronically stressed cancer cells (BxPC-3, HepG2, and SKOV-3) were treated with or without Res. Our results indicated that Res inhibited the proliferation and increased apoptosis in chronically stressed cancer cells.

Mechanistic studies have focused on Res chemoprevention and treatment of cancers, as well as on the effects of
Res on chronic diseases that may develop into cancer. The main pathways involved are the AMPK/Akt/mTOR (28), Wnt/ $\beta$-catenin (29), MAPK (30) and STAT3 (31) pathways. Ulcerative colitis is associated with a high risk of colon cancer, which is abated by Res treatment through downregulation of inflammatory stress (32). Epidemiological data have demonstrated that there is a strong association between diabetes mellitus and pancreatic cancer. Accumulating evidence has shown that the antidiabetic properties of Res may inhibit glucose uptake and suppress cancer cell metabolism $(30,33)$. It has been reported that the HBsAg prevalence for the Chinese population aged $1-59$ years was $7.2 \%$ (34), and chronic hepatitis B virus infection may promote the development of liver cancers, such as hepatoblastoma (35). However, the mechanism underlying the suppressive effect of Res on chronic stress-induced cancer has not been fully elucidated. In the present study, Res was used to treat chronically stressed cancer cells in order to demonstrate its anticancer effects on stress-induced cancer cells and to 
determine whether the underlying mechanism may involve inhibition of the ADRB-2-HIF-1 $\alpha$ axis (Fig. 5).

In conclusion, the findings of the present study demonstrated that Res can significantly inhibit the growth of tumor cells under chronic stress in a dose-dependent manner by suppressing their proliferation and promoting their apoptosis of tumor cells. The mechanism underlying these effects of Res may be mediated by inhibiting the activation of the ADRB-2-HIF-1 $\alpha$ axis in tumor cells under chronic stress. Thus, Res may be an effective treatment strategy for cancer patients who suffer from chronic stress.

\section{Acknowledgements}

Not applicable.

\section{Funding}

The present study was supported by grants from the National Natural Science Foundation of China (nos. 81402971 and 81702916).

\section{Availability of data and materials}

The datasets generated and analyzed in the present study are included in the published manuscript.

\section{Authors' contributions}

JM and MX designed the experiments, performed most of the experiments and drafted the manuscript; LC, WQ and SZ performed cell lines culture and analyzed the data; WD and XS designed the experiments and revised the manuscript. All authors read and approved the final manuscript.

\section{Ethics approval and consent to participate}

Not applicable.

\section{Patient consent for publication}

Not applicable.

\section{Competing interests}

All the authors declare that they have no competing interests to disclose.

\section{References}

1. Chen W, Zheng R, Baade PD, Zhang S, Zeng H, Bray F, Jemal A, Yu XQ and He J: Cancer statistics in China, 2015. CA Cancer J Clin 66: 115-132, 2016.

2. Chen W, Zheng R, Zhang S, Zeng H, Xia C, Zuo T, Yang Z, Zou X and He J: Cancer incidence and mortality in China, 2013. Cancer Lett 401: 63-71, 2017.

3. Massetti GM, Thomas CC, King J, Ragan K and Buchanan Lunsford N: Mental health problems and cancer risk factors among young adults. Am J Prev Med 53 (Issue 3, Suppl 1) S30-S39, 2017.

4. Shan T, Ma J, Ma Q, Guo K, Guo J, Li X, Li W, Liu J, Huang C, Wang $\mathrm{F}$, et al: $\beta 2-\mathrm{AR}-\mathrm{HIF}-1 \alpha$ : A novel regulatory axis for stress-induced pancreatic tumor growth and angiogenesis. Curr Mol Med 13: 1023-1034, 2013.
5. Xu Q, Zong L, Chen X, Jiang Z, Nan L, Li J, Duan W, Lei J, Zhang L, Ma J, et al: Resveratrol in the treatment of pancreatic cancer. Ann NY Acad Sci 1348: 10-19, 2015.

6. Vergara D, De Domenico S, Tinelli A, Stanca E, Del Mercato LL, Giudetti AM, Simeone P, Guazzelli N, Lessi M, Manzini C, et al: Anticancer effects of novel resveratrol analogues on human ovarian cancer cells. Mol Biosyst 13: 1131-1141, 2017.

7. Zeng YH, Zhou LY, Chen QZ, Li Y, Shao Y, Ren WY, Liao YP Wang H, Zhu JH, Huang M, et al: Resveratrol inactivates $\mathrm{PI} 3 \mathrm{~K} / \mathrm{Akt}$ signaling through upregulating BMP7 in human colon cancer cells. Oncol Rep 38: 456-464, 2017.

8. Yan Y, Zhou C, Li J, Chen K, Wang G, Wei G, Chen M and Li X: Resveratrol inhibits hepatocellular carcinoma progression driven by hepatic stellate cells by targeting Gli-1. Mol Cell Biochem 434: 17-24, 2017.

9. Saunier E, Antonio S, Regazzetti A, Auzeil N, Laprévote O, Shay JW, Coumoul X, Barouki R, Benelli C, Huc L, et al: Resveratrol reverses the Warburg effect by targeting the pyruvate dehydrogenase complex in colon cancer cells. Sci Rep 7: 6945 , 2017.

10. Bai Y, Yang H, Zhang G, Hu L, Lei Y, Qin Y, Yang Y, Wang Q, Li R and Mao Q: Inhibitory effects of resveratrol on the adhesion, migration and invasion of human bladder cancer cells. Mol Med Rep 15: 885-889, 2017.

11. Ferraresi A, Phadngam S, Morani F, Galetto A, Alabiso O, Chiorino $\mathrm{G}$ and Isidoro $\mathrm{C}$ : Resveratrol inhibits IL-6-induced ovarian cancer cell migration through epigenetic up-regulation of autophagy. Mol Carcinog 56: 1164-1181, 2017.

12. Deus CM, Serafim TL, Magalhães-Novais S, Vilaça A, Moreira AC, Sardão VA, Cardoso SM and Oliveira PJ: Sirtuin 1-dependent resveratrol cytotoxicity and pro-differentiation activity on breast cancer cells. Arch Toxicol 91: 1261-1278, 2017.

13. Yu Y, Zhao X, Zhang Y, Kang Y, Wang J and Liu Y: Antitumor activity of YM155, a selective survivin suppressant, in combination with cisplatin in hepatoblastoma. Oncol Rep 34: 407-414, 2015.

14. Kostev K, Jacob L and Kalder M: Risk of depression, anxiety, and adjustment disorders in women with a suspected but unconfirmed diagnosis of breast or genital organ cancer in Germany. Cancer Causes Control 28: 1021-1026, 2017.

15. Dzebo S, Mahmutovic J, Erkocevic H and Foco F: Frequency of depression and its correlation with quality of life of patients with oral cavity cancer. Mater Sociomed 29: 97-100, 2017.

16. Gonzalez-Saenz de Tejada M, Bilbao A, Baré M, Briones E, Sarasqueta C, Quintana JM and Escobar A; CARESS-CCR Group: Association between social support, functional status, and change in health-related quality of life and changes in anxiety and depression in colorectal cancer patients. Psychooncology 26: 1263-1269, 2017.

17. Jia Y, Li F, Liu YF, Zhao JP, Leng MM and Chen L: Depression and cancer risk: A systematic review and meta-analysis. Public Health 149: 138-148, 2017.

18. Vilardi BM, Bravo-Calderón DM, Bernabé DG, Oliveira SH and Oliveira DT: VEGF-C expression in oral cancer by neurotransmitter-induced activation of beta-adrenergic receptors. Tumour Biol 34: 139-143, 2013.

19. Huang XY, Wang HC, Yuan Z, Huang J and Zheng Q: Norepinephrine stimulates pancreatic cancer cell proliferation, migration and invasion via $\beta$-adrenergic receptor-dependent activation of P38/MAPK pathway. Hepatogastroenterology 59: 889-893, 2012.

20. Zhang D, Ma QY, Hu HT and Zhang M: $\beta 2$-adrenergic antagonists suppress pancreatic cancer cell invasion by inhibiting CREB, NFKB and AP-1. Cancer Biol Ther 10: 19-29, 2010.

21. Jiang Z, Chen X, Chen K, Sun L, Gao L, Zhou C, Lei M, Duan W, Wang Z, Ma Q, et al: YAP inhibition by resveratrol via activation of AMPK enhances the sensitivity of pancreatic cancer cells to gemcitabine. Nutrients 8: E546, 2016.

22. Tomas-Hernández S, Blanco J, Rojas C, Roca-Martínez J, Ojeda-Montes MJ, Beltrán-Debón R, Garcia-Vallve S, Pujadas G, Arola L and Mulero M: Resveratrol potently counteracts quercetin starvation-induced autophagy and sensitizes HepG2 cancer cells to apoptosis. Mol Nutr Food Res 62, 2018.

23. De Francesco EM, Maggiolini M and Musti AM: Crosstalk between Notch, HIF-1 $\alpha$ and GPER in Breast Cancer EMT. Int J Mol Sci 19: E2011, 2018.

24. Schito L and Semenza GL: Hypoxia-inducible factors: Master regulators of cancer progression. Trends Cancer 2: 758-770, 2016. 
25. Sun Y, Wang H, Liu M, Lin F and Hua J: Resveratrol abrogates the effects of hypoxia on cell proliferation, invasion and EMT in osteosarcoma cells through downregulation of the HIF-1 $\alpha$ protein. Mol Med Rep 11: 1975-1981, 2015.

26. Barbieri A, Bimonte S, Palma G, Luciano A, Rea D, Giudice A, Scognamiglio G, La Mantia E, Franco R, Perdonà S, et al: The stress hormone norepinephrine increases migration of prostate cancer cells in vitro and in vivo. Int J Oncol 47: 527-534, 2015.

27. Wang LP, Jin J, Lv FF, Cao J, Zhang J, Wang BY, Shao ZM, Hu XC and Wang ZH: Norepinephrine attenuates CXCR4 expression and the corresponding invasion of MDA-MB-231 breast cancer cells via $\beta 2$-adrenergic receptors. Eur Rev Med Pharmacol Sci 19: $1170-1181,2015$

28. Chang CH, Lee CY, Lu CC, Tsai FJ, Hsu YM, Tsao JW, Juan YN, Chiu HY, Yang JS and Wang CC: Resveratrol-induced autophagy and apoptosis in cisplatin-resistant human oral cancer CAR cells: A key role of AMPK and Akt/mTOR signaling. Int J Oncol 50: 873-882, 2017.

29. Reddivari L, Charepalli V, Radhakrishnan S, Vadde R, Elias RJ, Lambert JD and Vanamala JK: Grape compounds suppress colon cancer stem cells in vitro and in a rodent model of colon carcinogenesis. BMC Complement Altern Med 16: 278, 2016.
30. Cao L, Chen X, Xiao X, Ma Q and Li W: Resveratrol inhibits hyperglycemia-driven ROS-induced invasion and migration of pancreatic cancer cells via suppression of the ERK and p38 MAPK signaling pathways. Int J Oncol 49: 735-743, 2016.

31. Baek SH, Ko JH, Lee H, Jung J, Kong M, Lee JW, Lee J, Chinnathambi A, Zayed ME, Alharbi SA, et al: Resveratrol inhibits STAT3 signaling pathway through the induction of SOCS-1: Role in apoptosis induction and radiosensitization in head and neck tumor cells. Phytomedicine 23: 566-577, 2016.

32. Cui X, Jin Y, Hofseth AB, Pena E, Habiger J, Chumanevich A Poudyal D, Nagarkatti M, Nagarkatti PS, Singh UP and Hofseth LJ: Resveratrol suppresses colitis and colon cancer associated with colitis. Cancer Prev Res 3: 549-559, 2010.

33. León D, Uribe E, Zambrano A and Salas M: Implications of resveratrol on glucose uptake and metabolism. Molecules 22: E398, 2017

34. Liang X, Bi S, Yang W, Wang L, Cui G, Cui F, Zhang Y, Liu J, Gong X, Chen Y, et al: Reprint of: Epidemiological serosurvey of Hepatitis B in China - declining HBV prevalence due to Hepatitis B vaccination. Vaccine 31 (Suppl 9): J21-J28, 2013.

35. Casciano JC and Bouchard MJ: Hepatitis B virus X protein modulates cytosolic $\mathrm{Ca}^{2+}$ signaling in primary human hepatocytes. Virus Res 246: 23-27, 2018. 